\title{
Stability robustness of linear normal distributed parameter systems is $^{2}$
}

\author{
Shin-Hao Lu, I-Kong Fong * \\ Department of Electrical Engineering, National Taiwan University, Taipei, Taiwan 10617, ROC
}

Received 22 March 1999; received in revised form 1 August 2000; accepted 4 August 2000

\begin{abstract}
This paper considers the stability robustness analysis problem for linear distributed parameter systems containing known perturbation operators multiplied by uncertain parameters. The nominal system operators are assumed to be normal, but allowed to be unbounded. The perturbation operators are confined to some relative bounded set, but may be unbounded also. By using the Lyapunov stability criterion, simple bounds on uncertain parameters are derived to ensure the stability of the perturbed systems. Examples are provided to illustrate the usage of the theoretical results. (C) 2000 Elsevier Science B.V. All rights reserved.
\end{abstract}

Keywords: Distributed parameter system; Robust stability; Lyapunov method

\section{Introduction}

Distributed parameter systems (DPS) refer to systems whose dynamics are described by partial differential equations, delay equations, or functional differential equations defined on infinitedimensional spaces $[3,6,17]$. Thus, they are called the infinite-dimensional systems. Just like the finite-dimensional systems, DPS also have the stability and stability robustness problems. While there is a quite general theoretical framework [7,10] for treating finite-dimensional linear uncertain systems with structured or unstructured perturbations, the corresponding problem for DPS is much more difficult because of the intricacy of the underlying mathematics. This is particularly true, if simple and computable

\footnotetext{
This work is supported by the National Science Council of the Republic of China under Grant NSC 86-2213-E002-018.

* Corresponding author. Fax: +886-2-23660449.

E-mail address: ikfong@cc.ee.ntu.edu.tw (I-Kong Fong).
}

results are to be obtained. In the literature, many authors have, nevertheless, discussed the stability robustness problem of DPS with unstructured bounded perturbations [8], structured bounded perturbations [4], structured perturbations [18], and time-varying perturbations $[11,12]$.

In this paper, we consider via the Lyapunov stability approach a case of DPS with multiple structural perturbations, which consist of known perturbation operators multiplied by unknown constants [15]. The perturbations may appear in system dynamics as well as boundary conditions. For simplicity, it is assumed that the nominal system operators are normal, but possibly unbounded. In the mean time, the perturbation operators are required to be relative bounded [13] with respect to the nominal system operators, but may be unbounded also. We shall see from the provided example that these assumptions are not so restrictive.

Our development starts from the case with zero boundary condition, and comes to the first result when simple bounds on uncertain parameters are derived for 
the stability of the perturbed systems. Then, we move to systems with perturbations on the boundary conditions. We rely on the method devised in $[2,14]$, which transfers the original system into a suitable interpolation space, creates an equivalent system with zero boundary conditions and enables us to apply the established robust stability analysis method. Finally, we give examples to illustrate the usage of the theoretical results.

\section{Problem formulation}

Let $\mathscr{Z}_{1}$ and $\mathscr{Z}$ be two separable Hilbert spaces defined on a bounded domain $\Omega \subset \mathscr{R}^{m}$, where $m \geqslant 1$ is an integer, and $\mathscr{Z}_{1} \stackrel{\mathrm{d}}{\hookrightarrow} \mathscr{Z}$, i.e., $\mathscr{Z}_{1}$ is a dense subset of $\mathscr{Z}$. Consider a closed linear operator $A_{0}: \mathscr{D}\left(A_{0}\right) \rightarrow \mathscr{Z}$, which is densely defined on the domain $\mathscr{D}\left(\boldsymbol{A}_{0}\right) \subset \mathscr{Z}$. Assume that $\boldsymbol{A}_{0} \in \mathscr{L}\left(\mathscr{Z}_{1}, \mathscr{Z}\right)$, which means $\boldsymbol{A}_{0}$ is a linear bounded operator mapping from $\mathscr{Z}_{1}$ to $\mathscr{Z}$. Of course, $\mathscr{Z}_{1} \stackrel{\mathrm{d}}{\hookrightarrow} \mathscr{D}\left(\boldsymbol{A}_{0}\right)$. Moreover, consider a boundary operator $B$ defined on a Hilbert space $\partial Z$, which in turn is defined on the boundary of $\Omega$. Then, we focus on the following system with boundary condition $g(t)$ :

$\frac{\mathrm{d}}{\mathrm{d} t} z(t)=\boldsymbol{A}_{0} z(t)$

$B z(t)=g(t)$,

$z(0)=z_{0}, \quad z_{0} \in \mathscr{Z}$.

We say system (1) is exponentially stable if there exist positive numbers $\zeta$ and $\delta$ such that for all solution $z,\|z(t)\| \leqslant \zeta \mathrm{e}^{-\delta t}$, where $\|\cdot\|$ is the norm in $\mathscr{Z}$ (subsequently, $\|\cdot\|$ will also be used to represent the induced operator norm of operators defined on $\mathscr{Z}$, and the meaning will be clear from the context). For $g(t)=0$, we can define $A_{0}: \mathscr{D}\left(A_{0}\right) \rightarrow \mathscr{Z}$, the restriction of $\boldsymbol{A}_{0}$ to $\mathscr{D}\left(A_{0}\right)=\mathscr{D}\left(A_{0}\right) \cap \operatorname{Ker}(B)$, where $\operatorname{Ker}(B)$ is the kernel of $B$. If $A_{0}$ generates a $C_{0}$-semigroup $T_{0}(t)$ on $\mathscr{Z}$, then we can rewrite (1) as a standard Cauchy problem

$$
\frac{\mathrm{d}}{\mathrm{d} t} z(t)=A_{0} z(t)
$$

$z(0)=z_{0}, \quad z_{0} \in \mathscr{Z}$

for which the solution can be expressed as $z(t)=$ $T_{0}(t) z_{0}[3,6]$.

In this paper, for simplicity we restrict to the cases in which $\boldsymbol{A}_{0}$ is normal i.e., $\boldsymbol{A}_{0}$ satisfies $\boldsymbol{A}_{0}^{*} \boldsymbol{A}_{0}=\boldsymbol{A}_{0} \boldsymbol{A}_{0}^{*}$ [5]. In Section 3, we shall study the stability robustness of the system

$$
\begin{aligned}
& \frac{\mathrm{d}}{\mathrm{d} t} z(t)=\left(A_{0}+\sum_{i=1}^{i=\kappa} k_{i} A_{i}\right) z(t), \\
& z(0)=z_{0}, \quad z_{0} \in \mathscr{Z}
\end{aligned}
$$

with uncertain parameters $k_{i}$ 's and known perturbation operators $A_{i}$ 's. We require the perturbation operators $A_{i}$ 's to be relative bounded with respect to $A_{0}$ [13]. Note that this requirement does not restrict $A_{i}$ 's to be bounded operators. In Section 4, stability robustness problem of the more general case

$$
\begin{aligned}
& \frac{\mathrm{d}}{\mathrm{d} t} z(t)=\left(A_{0}+\sum_{i=1}^{i=\kappa} k_{i} A_{i}\right) z(t), \\
& B z(t)=k_{\kappa+1} G z(t), \\
& z(0)=z_{0}, \quad z_{0} \in \mathscr{Z}
\end{aligned}
$$

will be considered, with an additional bounded boundary perturbation operator $G$. Because the method devised in [2] will be applied in our derivation process, we need $\boldsymbol{A}_{0}$ to satisfy extra assumptions given therein, which guarantee that (4) can be transformed into a form like (3), but defined over certain interpolation spaces. These assumptions from [2] are not restrictive, as a wide class of interesting systems (such as Example 2 in Section 5 of this paper, or examples given in $[1,2])$ are known to satisfy them.

For (3) and (4), the robust stability problems assume that when all $k_{i}$ 's are zero, the nominal systems are exponentially stable. Letting $k=\left[k_{1} \cdots k_{k}\right]^{\mathrm{T}}$ for (3) and $k=\left[k_{1} \cdots k_{k+1}\right]^{\mathrm{T}}$ for (4), respectively, the problems are to find upper bounds $\bar{k}$ such that when $\|k\|_{2}=\sqrt{k^{\mathrm{T}} k}<\bar{k}$, the perturbed systems are still exponentially stable.

\section{Stability robustness with zero boundary condition}

First, we state the Lyapunov stability criterion for the DPS (2). We shall use $\mathscr{L}(\mathscr{Z})$ as a shorthand notation for $\mathscr{L}(\mathscr{Z}, \mathscr{Z})$, and $P>0$ to denote that the operator $P$ is positive definite. Also, we say an operator $Q$ is coercive if there exists a $\varepsilon_{Q}>0$ such that $\langle Q z, z\rangle \geqslant \varepsilon_{Q}\langle z, z\rangle$ for all $z \in \mathscr{Z}$, where $\langle\cdot, \cdot\rangle$ is the inner product in $\mathscr{Z}$. 
Theorem 1 (Curtain and Zwart [6], Gohberg et al. [9]). If there exist a $P_{0}>0$ in $\mathscr{L}(\mathscr{Z})$ and a coercive $Q_{0}$ in $\mathscr{L}(\mathscr{Z})$ such that

$\left\langle P_{0} z, A_{0} z\right\rangle+\left\langle A_{0} z, P_{0} z\right\rangle=-\left\langle Q_{0} z, z\right\rangle$

for all $z \in \mathscr{D}\left(A_{0}\right)$, then (2), or simply $A_{0}$, is exponentially stable.

In [16] it is noted that if general perturbations are to be treated by the Lyapunov stability criterion, then the nominal DPS must be of linear parabolic type. But here we consider only linear perturbations, therefore, the perturbed DPS is also linear and the Lyapunov stability criterion stated in Theorem 1 can be applied. Moreover, it may be deduced [6] that if $A_{0}$ is exponentially stable, then any coercive $Q_{0}$ and $P_{0}$ defined by $P_{0} z=\int_{0}^{\infty} T_{0}^{*}(t) Q_{0} T_{0}(t) z \mathrm{~d} t$ satisfy the conditions required by Theorem 1 , where $T_{0}^{*}$ means the adjoint operator of $T_{0}$.

From the normality assumption of $\boldsymbol{A}_{0}$, we know that $A_{0}$ is also normal. Then we have the following results.

Theorem 2. Let $A_{0}$ be normal and exponentially stable. Assume that $\mathscr{D}\left(A_{0}\right)=\mathscr{D}\left(A_{0}+A_{0}^{*}\right)$, then, we have: (i) $\left(A_{0}+A_{0}^{*}\right): \mathscr{D}\left(A_{0}\right) \subset \mathscr{Z} \rightarrow \mathscr{Z}$ is the infinitesimal generator of $T_{0}^{*}(t) T_{0}(t)$, and $A_{0}+A_{0}^{*}$ is exponentially stable. (ii) Define $P_{0}$ as $P_{0} z=\int_{0}^{\infty} T_{0}^{*}(t) T_{0}(t) z \mathrm{~d} t$, then $P_{0} z \in \mathscr{D}\left(A_{0}\right)=\mathscr{D}\left(A_{0}^{*}\right),\left(A_{0}+A_{0}^{*}\right) P_{0} z=-z, A_{0} P_{0} \in$ $\mathscr{L}(\mathscr{Z})$, and $A_{0}^{*} P_{0} \in \mathscr{L}(\mathscr{Z})$.

Proof. (i) Firstly, for $\lambda \in \rho\left(A_{0}\right)$, the resolvent set of $A_{0}$, let $R(\lambda) z=\left(\lambda I-A_{0}\right)^{-1} z$. Since $A_{0}^{*} A_{0}=A_{0} A_{0}^{*}$ implies $\left(\lambda I-A_{0}\right) A_{0}^{*} R(\lambda)=A_{0}^{*}\left(\lambda I-A_{0}\right) R(\lambda)=A_{0}^{*}$, we have $A_{0}^{*} R(\lambda)=R(\lambda) A_{0}^{*}$. Secondly, it is easy to check that $A_{\lambda}^{0}=\lambda^{2} R(\lambda)-\lambda I \in \mathscr{L}(\mathscr{Z})$ and $A_{\lambda}^{0} A_{0}^{*}=A_{0}^{*} A_{\lambda}^{0}$. Hence $T_{0}(t) A_{0}^{*} z=\lim _{\lambda \rightarrow \infty} \mathrm{e}^{t A_{\lambda}^{0}} A_{0}^{*} z=$ $A_{0}^{*} \lim _{\lambda \rightarrow \infty} \mathrm{e}^{t A_{\lambda}^{0}}=A_{0}^{*} T_{0}(t) z$, or $T_{0}(t) A_{0}^{*}=A_{0}^{*} T_{0}(t)$. Thirdly, for $z \in \mathscr{D}\left(A_{0}\right)$, consider the differentiable function $\tau \mapsto T_{0}^{*}(t-\tau) T_{0}(t-\tau) T_{0}(\tau) T_{0}^{*}(\tau) z$. The equality $T_{0}(t) A_{0}^{*}=A_{0}^{*} T_{0}(t)$ implies that

$$
\begin{aligned}
\frac{\mathrm{d}}{\mathrm{d} \tau}\{ & {\left.\left[T_{0}^{*}(t-\tau) T_{0}(t-\tau)\right]\left[T_{0}(\tau) T_{0}^{*}(\tau)\right]\right\} } \\
= & T_{0}^{*}(t-\tau)\left[-\left(A_{0}^{*}+A_{0}\right) T_{0}(t-\tau) T_{0}(\tau)\right. \\
& \left.+T_{0}(t-\tau) T_{0}(\tau)\left(A_{0}^{*}+A_{0}\right)\right] T_{0}^{*}(\tau) \\
= & 0 .
\end{aligned}
$$

Therefore, $\tau \mapsto T_{0}^{*}(t-\tau) T_{0}(t-\tau) T_{0}(\tau) T_{0}^{*}(\tau) z$ is constant, and in particular its values at $\tau=0$ and $\tau=t$ are the same. Thus, $T_{0}^{*}(t) T_{0}(t)=T_{0}(t) T_{0}^{*}(t)$. Finally, if $T_{0}^{*}(t) T_{0}(t)=T_{0}(t) T_{0}^{*}(t)$, then by direct check we can conclude that $T_{0}^{*} T_{0}$ is a $C_{0}$-semigroup with the infinitesimal generator $A_{0}+A_{0}^{*}$. With this result, it is clear that the exponential stability of $A_{0}$ implies that of $A_{0}+A_{0}^{*}$.

(ii) For exponentially stable $A_{0}$, it is easy to show that $P_{0}$ is bounded. Since $A_{0}+A_{0}^{*}$ is the infinitesimal generator of $T_{0}^{*}(t) T_{0}(t), \int_{0}^{t} T_{0}^{*}(s) T_{0}(s) z \mathrm{~d} s \in \mathscr{D}\left(A_{0}+\right.$ $A_{0}^{*}$ ) for all $t>0$ [6]. By the normality of $A_{0}$ and the assumption, we have $\mathscr{D}\left(A_{0}\right)=\mathscr{D}\left(A_{0}^{*}\right)=\mathscr{D}\left(A_{0}+A_{0}^{*}\right)$ and $\left(A_{0}+A_{0}^{*}\right) \int_{0}^{\infty} T_{0}^{*}(s) T_{0}(s) z \mathrm{~d} s=-z$. Also note that since $P_{0} z \in \mathscr{D}\left(A_{0}\right), A_{0} P_{0} z$ is well defined for all $z \in$ $\mathscr{Z}$. With the closeness of $A_{0}$ and $A_{0} P_{0}$, by the closed graph theorem [5] we know that $A_{0} P_{0}$ is bounded. The same argument also applies to $A_{0}^{*}$.

As to the perturbation operators $A_{i}$ 's, for simplicity we confine our discussion to those belonging to the set $\mathscr{P}\left(A_{0}\right)$ defined below.

Definition 1. The set of relative bounded perturbations with respect to $A_{0}$ is defined as $\mathscr{P}\left(A_{0}\right)=$ $\left\{A: \mathscr{D}(A) \subset \mathscr{Z} \rightarrow \mathscr{Z} \mid \mathscr{D}\left(A_{0}\right) \subset \mathscr{D}(A)\right.$, and there exist nonnegative numbers $\alpha, \beta$ such that $\|A z\| \leqslant \alpha\left\|A_{0} z\right\|+$ $\left.\beta\|z\| \forall z \in \mathscr{D}\left(A_{0}\right)\right\}$.

Note that in this definition $A_{0}$ is allowed to be unbounded, and $\mathscr{L}(\mathscr{Z}) \subset \mathscr{P}\left(A_{0}\right)$. A rich amount of examples of relative bounded operators can be found in [13].

Under these postulates, the following theorem is the main result of this section.

Theorem 3. Suppose in system (3) $A_{0}$ is normal, exponentially stable, and the infinitesimal generator of $T_{0}(t)$. Assume that $\mathscr{D}\left(A_{0}\right)=\mathscr{D}\left(A_{0}+A_{0}^{*}\right)$, for $i=1, \ldots, \kappa$ the perturbation operator $A_{i}$ satisfies $A_{i}^{*} \in \mathscr{P}\left(A_{0}^{*}\right)$, and $\left(A_{0}+\sum_{i=1}^{\kappa} k_{i} A_{i}\right): \mathscr{D}\left(A_{0}\right) \subset \mathscr{Z} \rightarrow \mathscr{Z}$ generates a $C_{0}$-semigroup $T_{\kappa}(t)$. Let $P_{0}$ be defined as $P_{0} z=$ $\int_{0}^{\infty} T_{0}^{*}(t) T_{0}(t) z \mathrm{~d} t$, and

$\bar{k}_{i}=\inf _{\|z\|=1} \frac{1}{\left|\left\langle\left(P_{0} A_{i}+A_{i}^{*} P_{0}\right) z, z\right\rangle\right|}$.

If the uncertain parameters are limited to $\|k\|_{2}<\bar{k}=$ $\left(\sum_{i=1}^{\kappa} \bar{k}_{i}^{-2}\right)^{-1 / 2}$, then system (3) is exponentially stable.

Proof. Clearly $P_{0}>0$, and by Theorem $2 P_{0}$ satisfies $\left\langle A_{0} z, P_{0} z\right\rangle+\left\langle P_{0} z, A_{0} z\right\rangle=-\langle z, z\rangle$ for all $z \in$ $\mathscr{D}\left(A_{0}\right)$. Let the Lyapunov function candidate be $\ell(t, z)=\left\langle P_{0} T_{\kappa}(t) z, T_{\kappa}(t) z\right\rangle$ for all $z \in \mathscr{D}\left(A_{0}\right)$, where 
$T_{\kappa}(t) z \in \mathscr{D}\left(A_{0}+\sum_{i=1}^{\kappa} k_{i} A_{i}\right)=\mathscr{D}\left(A_{0}\right)$. From direct computation, we get

$$
\frac{\mathrm{d} \ell}{\mathrm{d} t}=-\left\langle\left[I-\sum_{i=1}^{\kappa} k_{i}\left(P_{0} A_{i}+A_{i}^{*} P_{0}\right)\right] T_{\kappa}(t) z, T_{\kappa}(t) z\right\rangle .
$$

Since $A_{i}^{*} \in \mathscr{P}\left(A_{0}^{*}\right)$ and for all $z \in \mathscr{Z}, P_{0} z \in \mathscr{D}\left(A_{0}\right)=$ $\mathscr{D}\left(A_{0}^{*}\right), A_{i}^{*} P_{0}$ is well defined for all $z \in \mathscr{Z}$. Furthermore, $A_{i}^{*} \in \mathscr{P}\left(A_{0}^{*}\right)$ means there exist nonnegative $\alpha_{i}, \beta_{i}$ such that $\left\|A_{i}^{*} P_{0} z\right\| \leqslant\left(\alpha_{i}\left\|A_{0}^{*} P_{0}\right\|+\beta_{i}\left\|P_{0}\right\|\right)\|z\|$, which together with the boundedness of $A_{0}^{*} P_{0}$ and $P_{0}$ imply that $A_{i}^{*} P_{0} \in \mathscr{L}(\mathscr{Z})$. This, in turn, implies that $\left(A_{i}^{*} P_{0}\right)^{*} \in \mathscr{L}(\mathscr{Z})$, and that $P_{0} A_{i}$ has a bounded extension defined on $\mathscr{Z}$, because $P_{0} A_{i}$ is a restriction of $\left(A_{i}^{*} P_{0}\right)^{*}$. We use the same notation $P_{0} A_{i}$ to denote this extension. Thus, we have $P_{0} A_{i} \in \mathscr{L}(\mathscr{Z})$ and $\left\|A_{i}^{*} P_{0}\right\|=\left\|P_{0} A_{i}\right\|$.

Next, we want to determine a range of $k$ within which $I-\sum_{i=1}^{\kappa} k_{i}\left(P_{0} A_{i}+A_{i}^{*} P_{0}\right)$ is coercive. Define a set $\mathscr{K}(z)=\left\{k \mid \sum_{i=1}^{\kappa}\left\langle\left(P_{0} A_{i}+A_{i}^{*} P_{0}\right) z, z\right\rangle k_{i}<\langle z, z\rangle\right\}$. Note that for each nonzero $z \in \mathscr{Z}, \mathscr{K}(z)$ is a half-space in $\mathscr{R}^{\kappa}$ which includes the origin of $\mathscr{R}^{\kappa}$. Also, the boundary of $\mathscr{K}(z)$ is a hyperplane which intercepts the $i$ th coordinate axis of $\mathscr{R}^{\kappa}$ at $k_{i}(z)=\|z\|^{2} /\left\langle\left(P_{0} A_{i}+A_{i}^{*} P_{0}\right) z, z\right\rangle$ and has the minimum distance $\left[\sum_{i=1}^{\kappa} k_{i}^{-2}(z)\right]^{-1 / 2}$ from the origin of $\mathscr{R}^{\kappa}$. Here, when necessary, we take $\|z\|^{2} / 0=\infty$ and $\infty^{-2}=0$. Let $\bar{k}_{i}=\inf _{\|z\|=1}\left|k_{i}(z)\right|$. The infimum exists and is positive because both $P_{0} A_{i}$ and $A_{i}^{*} P_{0}$ are in $\mathscr{L}(\mathscr{Z})$. It is clear that if $\|k\|_{2}<\left(\sum_{i=1}^{\kappa} \bar{k}_{i}^{-2}\right)^{-1 / 2}$, then $k \in \mathscr{K}(z)$ for all $z \in \mathscr{Z}$. This is the range we are looking for, which, by the Lyapunov stability criterion, is also a range of uncertain parameters for the robust stability of system (3).

To utilize the theorem, the main difficulty is the search of the infimum in (6) in order to get a good bound. In fact, it is not always feasible, so we give the following two corollaries to handle some special cases.

Corollary 1. Under the assumptions of Theorem 3, if we know the relative bounded coefficients $\alpha_{i}$ and $\beta_{i}$ such that $\left\|A_{i}^{*} z\right\| \leqslant \alpha_{i}\left\|A_{0}^{*} z\right\|+\beta_{i}\|z\|$ for all $z \in \mathscr{D}\left(A_{0}\right)$, as well as the positive constants $\mu$ and $\omega$ such that $\left\|T_{0}(t)\right\| \leqslant \mu \mathrm{e}^{-\omega t}$ for all $t \geqslant 0$, then

$\bar{k}_{i} \geqslant \frac{1}{2\left(1+r_{0}\right) \alpha_{i}+\left(\mu^{2} / \omega\right) \beta_{i}}$

where $r_{0}=\left\|A_{0} P_{0}\right\|$.
Proof. Since

$$
\begin{aligned}
\left\|A_{i}^{*} P_{0} z\right\| & \leqslant \alpha_{i}\left\|A_{0}^{*} P_{0} z\right\|+\beta_{i}\left\|P_{0} z\right\| \\
& =\alpha_{i}\left\|-A_{0} P_{0} z-z\right\|+\beta_{i}\left\|P_{0} z\right\| \\
& \leqslant\left[\alpha_{i}\left(1+r_{0}\right)+\beta_{i} \frac{\mu^{2}}{2 \omega}\right]\|z\|,
\end{aligned}
$$

we have

$$
\begin{aligned}
& \left|\left\langle\left(P_{0} A_{i}+A_{i}^{*} P_{0}\right) z, z\right\rangle\right| \\
& \quad \leqslant\left\|\left(P_{0} A_{i}+A_{i}^{*} P_{0}\right)\right\| \cdot\|z\|^{2} \leqslant 2\left\|A_{i}^{*} P_{0}\right\| \cdot\|z\|^{2} \\
& \quad \leqslant\left[2 \alpha_{i}\left(1+r_{0}\right)+\beta_{i} \frac{\mu^{2}}{\omega}\right]\|z\|^{2} .
\end{aligned}
$$

Hence,

$$
\frac{1}{\left|\left\langle\left(P_{0} A_{i}+A_{i}^{*} P_{0}\right) z, z\right\rangle\right|} \geqslant \frac{1}{2 \alpha_{i}\left(1+r_{0}\right)+\beta_{i}\left(\mu^{2} / \omega\right)}
$$

By the definition of $\bar{k}_{i}$, we have $\bar{k}_{i} \geqslant \frac{1}{2\left(1+r_{0}\right) \alpha_{i}+\left(\mu^{2} / \omega\right) \beta_{i}}$.

Corollary 2. Under the assumptions of Corollary 1, if $A_{0}=A_{0}^{*}$ is self-adjoint, then

$\bar{k}_{i} \geqslant \frac{1}{\alpha_{i}+(\mu / \omega) \beta_{i}}$.

Proof. For $A_{0}=A_{0}^{*}$, we have $T_{0}^{*}=T_{0}$ because $\mathscr{Z}$ is a Hilbert space. Thus,

$$
\begin{aligned}
P_{0} z & =\int_{0}^{\infty} T_{0}^{*}(t) T_{0}(t) z \mathrm{~d} t=\int_{0}^{\infty} T_{0}(t) T_{0}(t) z \mathrm{~d} t \\
& =\int_{0}^{\infty} T_{0}(2 t) z \mathrm{~d} t=\frac{1}{2} \int_{0}^{\infty} T_{0}(t) z \mathrm{~d} t
\end{aligned}
$$

$P_{0} z \in \mathscr{D}\left(A_{0}\right)=\mathscr{D}\left(A_{0}^{*}\right)$, and $A_{0} P_{0} z=-\frac{1}{2} z$. Note that

$\left\|A_{i}^{*} P_{0} z\right\| \leqslant \alpha_{i}\left\|A_{0}^{*} P_{0} z\right\|+\beta_{i}\left\|P_{0} z\right\|=\left(\frac{\alpha_{i}}{2}+\beta_{i} \frac{\mu}{2 \omega}\right)\|z\|$.

Therefore,

$\left|\left\langle\left(P_{0} A_{i}+A_{i}^{*} P_{0}\right) z, z\right\rangle\right| \leqslant\left(\alpha_{i}+\frac{\mu}{\omega} \beta_{i}\right)\|z\|$.

The rest of the proof is similar to that in Corollary 1 .

The result of Corollary 2 simplifies the computation for self-adjoint systems, such as those described by the heat conduction or diffusion equations.

\section{Stability robustness with boundary perturbation}

To extend the results derived in Section 3 to system (4), we adopt the mathematical framework devised 
by Amann [2]. To do so, we must restrict our discussion to parabolic type evolution problems on Hilbert space only, and need to assume that $A_{0}$ and $B$ in (1) satisfy the assumptions (A1)-(A7) made in [2]. In these seven assumptions, the first three give a detailed characterization of the domains of $\boldsymbol{A}_{0}$ and its adjoint operator, which limit the types of parabolic equations that can be handled. The fourth assumption defines the boundary space of the boundary operator $B$, and the fifth assumption ensures that the Green's formula for $A_{0}$ and $B$ is valid. The sixth one poses the conditions for deriving $A_{0}$, which is the restriction of $\boldsymbol{A}_{0}$ to the kernel of $B$, and the last one is $\rho\left(A_{0}\right) \cap \rho\left(A_{0}^{*}\right) \neq$ $\emptyset$. These assumptions are basic ones to ensure that the derivations based on the Green's formula can be done. In Section 5, Example 2 gives a case that fits into such a framework, and more examples can be found in $[1,2]$. Subject to these basic assumptions, the method developed in [2] enables us to construct the "scale" $\left\{\left(\mathscr{E}_{a}, A_{0}^{a}\right) ; a \in \mathscr{R}\right\}$ with $\mathscr{E}_{0}=\mathscr{Z}$. Here we require every $\mathscr{E}_{a}$ to be a Hilbert space with norm $\|\cdot\|_{a}$ and inner product $\langle\cdot, \cdot\rangle_{a}$. Also we use $T_{0}^{a}$ to denote the $C_{0}$-semigroup generated by $A_{0}^{a}$. From [2] it is known that there exist a real number $b \in(0,1)$, an operator $R_{b}$, and a real number $\sigma \in \rho\left(A_{0}\right)$ such that, in the sense of weak solution, the solution of (1) is the same as the wild solution of

$\frac{\mathrm{d}}{\mathrm{d} t} z(t)=A_{0}^{b-1} z(t)+\left(\sigma I-A_{0}^{b-1}\right) R_{b} g(t)$,

$z(0)=z_{0}$

in the interpolation space $E_{b-1}$. Note that if we consider time-invariant boundary operator $B$ and bounded boundary condition function $g(\cdot)$, then the solution of (10) is a global solution [2] which preserves the stability property of the solution of (1).

For $g(t)=k_{\kappa+1} G z(t)$, if $R_{b} G_{b} \in \mathscr{L}\left(\mathscr{E}_{b}\right)$, where $G_{b}$ is the restriction of $G$ in $\mathscr{E}_{b}$, then we have

$$
\begin{aligned}
& \left\|\left[\left(\sigma I-A_{0}^{b-1}\right) R_{b} G_{b}\right]^{*} z\right\|_{b-1} \\
& \quad \leqslant\left\|R_{b} G_{b}\right\|_{b} \cdot\left[\left\|\left(A_{0}^{b-1}\right)^{*} z\right\|_{b-1}+|\sigma| \cdot\|z\|_{b-1}\right]
\end{aligned}
$$

for all $z \in \mathscr{E}_{b-1}$, which means that $\left[\left(\sigma I-A_{0}^{b-1}\right)\right.$ $\left.R_{b} G_{b}\right]^{*} \in \mathscr{P}\left[\left(A_{0}^{b-1}\right)^{*}\right]$ with relative bounded coefficients $\alpha_{\kappa+1}^{b-1}=\left\|R_{b} G_{b}\right\|_{b}$, and $\beta_{\kappa+1}^{b-1}=|\sigma| \cdot\left\|R_{b} G_{b}\right\|_{b}$. Furthermore, assume the perturbation operators $A_{i}$, $i=1, \ldots, \kappa$, can be extended to $\mathscr{E}_{b-1}$ to be $A_{i}^{b-1}$, and we can transform the perturbed system (4) to

$$
\begin{aligned}
\frac{\mathrm{d}}{\mathrm{d} t} z(t)= & \left(A_{0}^{b-1}+\sum_{i=1}^{\kappa} k_{i} A_{i}^{b-1}\right) z(t) \\
& +k_{\kappa+1}\left(\sigma I-A_{0}^{b-1}\right) R_{b} G_{b} z(t)
\end{aligned}
$$

$z(0)=z_{0}$.

Under the above setting, we can apply the result obtained in Section 3 to system (11) for solving the robust stability problem of system (4). This is done in the following theorem and corollary.

Theorem 4. Suppose in the system (11) $A_{0}^{b-1}$ is exponentially stable as well as normal in $\mathscr{E}_{b-1}$, and $\left\|T_{0}^{b-1}(t)\right\|_{b-1} \leqslant \mu \mathrm{e}^{-\omega t}$ for some positive $\mu$, $\omega$, and all $t \geqslant 0$. Assume that $\mathscr{D}\left(A_{0}^{b-1}\right)=\mathscr{D}\left[A_{0}^{b-1}+\left(A_{0}^{b-1}\right)^{*}\right]$, $\left(A_{i}^{b-1}\right)^{*} \in \mathscr{P}\left[\left(A_{0}^{b-1}\right)^{*}\right]$ and $\left[A_{0}^{b-1}+\sum_{i=1}^{\kappa} k_{i} A_{i}^{b-1}+\right.$ $\left.k_{\kappa+1}\left(\sigma I-A_{0}^{b-1}\right) R_{b} G_{b}\right]: \mathscr{D}\left(A_{0}^{b-1}\right) \subset \mathscr{E}_{b-1} \rightarrow \mathscr{E}_{b-1}$ generates a $C_{0}$-semigroup $T_{\kappa+1}^{b-1}(t)$ on $\mathscr{E}_{b-1}$. Let $P_{0}^{b-1}$ be defined by $P_{0}^{b-1} z=\int_{0}^{\infty}\left(T_{0}^{b-1}\right)^{*}(t) T_{0}^{b-1}(t) z \mathrm{~d} t$, and $\begin{aligned} \bar{k}_{i}= & \inf _{\|z\|=1} \frac{1}{\left|\left\langle\left[P_{0}^{b-1} A_{i}^{b-1}+\left(A_{i}^{b-1}\right)^{*} P_{0}^{b-1}\right] z, z\right\rangle_{b-1}\right|}, \\ & i=1, \ldots, \kappa,\end{aligned}$ $\bar{k}_{\kappa+1}=\frac{1}{2\left(1+r_{0}^{b-1}\right) \alpha_{\kappa+1}^{b-1}+\left(\mu^{2} / \omega\right) \beta_{\kappa+1}^{b-1}}$,

where $r_{0}^{b-1}=\left\|A_{0}^{b-1} P_{0}^{b-1}\right\|_{b-1}$. If $\|k\|_{2}<\bar{k}=$ $\left(\sum_{i=1}^{\kappa+1} \bar{k}_{i}^{-2}\right)^{-1 / 2}$, then the perturbed system (4) is exponentially stable.

Proof. The proof is the same as that for Theorem 3, except the space considered here is $\mathscr{E}_{b-1}$.

Corollary 3. Under the assumptions of Theorem 4, if $A_{0}^{b-1}=\left(A_{0}^{b-1}\right)^{*}$, then

$\bar{k}_{i} \geqslant \frac{1}{\alpha_{i}^{b-1}+(\mu / \omega) \beta_{i}^{b-1}}, \quad i=1, \ldots, \kappa$,

where $\alpha_{i}^{b-1}$ and $\beta_{i}^{b-1}$ are the relative bounded coefficients of perturbation operator $\left(A_{i}^{b-1}\right)^{*}$ with respect to $\left(A_{0}^{b-1}\right)^{*}$.

\section{Examples}

Example 1. Consider the distributed parameter system described by the following partial differential 
equation, which is basically a diffusion equation defined on $\mathscr{Z}=\mathscr{H}(0,1)=\left\{\left.f(x)\left|\int_{0}^{1}\right| f(x)\right|^{2} \mathrm{~d} x<\infty\right\}$ :

$$
\begin{aligned}
\frac{\partial}{\partial t} z= & \left(\frac{\partial^{2}}{\partial x^{2}}+\gamma I\right) z+k_{1} \frac{\partial}{\partial x} z \\
& +k_{2} \int_{0}^{1} h(x) z(t, x) \mathrm{d} x \cdot v(x),
\end{aligned}
$$

$z(t, 0)=z(t, 1)=0$,

$z(0, x)=z_{0}$,

where $h(x) \geqslant 0$ for all $x \in[0,1],\|h(x)\|=1,\|v(x)\|=1$, and $\gamma<0$. Putting this system into the frame of our discussion, we have $A_{0} z=\left(\partial^{2} / \partial x^{2}+\gamma I\right) z$ with $\mathscr{D}\left(A_{0}\right)=$ $\left\{z \in \mathscr{Z} \mid z\right.$ and $\partial z / \partial x$ absolutely continuous, $\partial^{2} z / \partial x^{2} \in$ $\mathscr{Z}, z(0)=z(1)=0\}$. It is easy to verify that $A_{0}^{*}=A_{0}$, i.e., $A_{0}$ is self-adjoint, and $T_{0}(t)$ satisfies $\left\|T_{0}(t)\right\| \leqslant \mathrm{e}^{\gamma t}$. The perturbation operators are $A_{1} z=(\partial / \partial x) z$ with $\mathscr{D}\left(A_{1}\right)=$ $\{z \in \mathscr{Z} \mid z$ absolutely continuous, $\partial z / \partial x \in \mathscr{Z}, z(0)=$ $z(1)=0\}$ and $A_{2} z=\int_{0}^{1} h(x) z(t, x) \mathrm{d} x \cdot v(x)$.

First, we check the condition of the perturbation operator $A_{1}$. Note that $A_{1}^{*}=-A_{1}$. From [13] we have the following estimate for all $z \in \mathscr{Z}$ :

$\left\|\frac{\partial z}{\partial x}\right\| \leqslant \frac{1}{n-1}\left\|\frac{\partial^{2} z}{\partial x^{2}}\right\|+\frac{2 n(n+1)}{(n-1)}\|z\|$,

where $n$ is any integer larger than unity, i.e., we have $\left\|A_{1}^{*} z\right\| \leqslant \frac{1}{n-1}\left\|A_{0}^{*} z\right\|+\frac{2 n(n+1)}{(n-1)}\|z\|$

and $\mathscr{D}\left(A_{0}^{*}\right) \subset \mathscr{D}\left(A_{1}^{*}\right)$. Thus, $A_{1}^{*} \in \mathscr{P}\left(A_{1}^{*}\right)$ with relative bounded coefficients $\alpha_{1}=1 /(n-1)$ and $\beta_{1}=2 n(n+$ $1) /(n-1)$. By (9) a lower bound of $\bar{k}_{1}$ can be obtained as

$\bar{k}_{1}=\frac{1}{\alpha_{1}+(\mu / \omega) \beta_{1}}=\frac{|\gamma|(n-1)}{|\gamma|+2 n(n+1)}$.

For the perturbation operator $A_{2}$, since $\|h(x)\|=$ $\|v(x)\|=1$, we have $A_{2} \in \mathscr{L}(\mathscr{Z})$ and $A_{2}^{*} \in \mathscr{L}(\mathscr{Z})$. Thus, the relative bounded coefficients $\alpha_{2}=0$ and $\beta_{2}=1$ can be used.

Finally, we give the results of $\bar{k}_{1}, \bar{k}_{2}$, and $\bar{k}$ corresponding to various values of $\gamma$ and $n$ in Table 1

Example 2. This example follows the example 1 given in [1]. Let $\Omega$ be a bounded domain in $\mathscr{R}^{n}$ with boundary $\partial \Omega, s \in \mathscr{R}, \mathscr{H}^{0}=\mathscr{L}_{2}\left(\Omega, \mathscr{C}^{N}\right)$ be the space of Lebesgue square integrable functions, $\mathscr{H}^{s}=\mathscr{W}_{2}^{s}\left(\Omega, \mathscr{C}^{N}\right)$ be the Sobolev space of order $s$, and
Table 1

\begin{tabular}{llrl}
\hline$\gamma, n$ & $\bar{k}_{1}$ & $\bar{k}_{2}$ & $\bar{k}$ \\
\hline$\gamma=-1, n=3$ & 0.08 & 1 & 0.0797 \\
$\gamma=-2, n=3$ & 0.1538 & 2 & 0.1533 \\
$\gamma=-10, n=4$ & 0.6 & 10 & 0.5989 \\
\hline
\end{tabular}

$\partial \mathscr{H}^{s}=\mathscr{W}_{2}^{s}\left(\partial \Omega, \mathscr{C}^{N}\right)$. Consider the system described by

$\frac{\partial}{\partial t} z=A_{0} z$

$B z=k_{1} G z$,

$z(0)=z_{0}$,

where $z=\left[z_{1}(t, x) \cdots z_{N}(t, x)\right]^{\mathrm{T}}, x=\left[x_{1} \cdots x_{n}\right]^{\mathrm{T}}$, $\boldsymbol{A}_{0}=D \Delta+\gamma I: \mathscr{H}^{2} \rightarrow \mathscr{H}^{0}, D=\operatorname{diag}\left[d_{1}, \ldots, d_{N}\right]$ with $d_{1}>0, \Delta$ is the Laplacian, $B=D \frac{\partial}{\partial v}$ with $v$ being the outward normal vector on the boundary, $k_{1} \in \mathscr{R}$ is an uncertain parameter, and $G z=\left[h_{1}(x) \int_{Q}\right.$ $\left.g_{1}(x) z_{1}(t, x) \mathrm{d} x \cdots h_{N}(x) \int_{\Omega} g_{N}(x) z_{N}(t, x) \mathrm{d} x\right]^{\mathrm{T}} \quad$ with given functions $h_{i}(x)$ and $g_{i}(x)$.

Let $\mathscr{W}_{B}^{\prime}=\left\{z \in \mathscr{H}^{2} \mid B z=0\right\}, D \Delta_{B}$ be the operator $D \Delta$ restricted to $\mathscr{W}_{B}^{\prime}$, and $A_{0}=D \Delta_{B}+\gamma I$. Following the result of Section 4 and assuming that $\gamma$ makes $A_{0}$ exponentially stable, we know that the stability robustness problem of system (14) with respect to the uncertain parameter $k_{1}$ is equivalent to that of the system

$\frac{\partial}{\partial t} z(t)=A_{0}^{b-1} z(t)+k_{1}\left(\sigma-A_{0}^{b-1}\right) R_{b} G_{b} z(t)$,

$z(0)=z_{0}$,

where $R_{b}=\left.\left(A_{0}, B\right)^{-1}\right|_{\{0\} \times \partial \mathscr{H}^{2 b-3 / 2}}$ [2] for some $0 \leqslant b \leqslant 1$, the operators $A_{0}^{b-1}, G_{b}$ are as explained in Section 4 , and $\sigma$ can be chosen to be zero because here $A_{0}$ is basically an exponentially stable Laplacian. From [2] it is known that there exists a constant $\eta$ depending on $b$ such that $\left\|R_{b}\right\| \leqslant \eta$. Therefore, the robustness problem of the above system can be treated by Corollary 3 , and the relative bounded coefficients are $\alpha_{1}^{b-1}=\eta \cdot\left\|G_{b}\right\|$ and $\beta_{1}^{b-1}=0$, where $\left\|G_{b}\right\|$ is determined by the functions $h_{i}(x)$ and $g_{i}(x)$.

\section{Conclusion}

The robust stability problem of nominally normal DPS is discussed via the Lyapunov approach. The 
perturbed system may contain a class of unbounded perturbation operators multiplied by uncertain parameters. Bounds on these uncertain parameters appearing in system dynamics as well as boundary conditions are derived to ensure the stability of the perturbed system. The results may be more conservative compared with those for finite dimensional systems, but for DPS such simple and computable results are harder to find. It is hoped that in the future more improved results in this regard will appear.

\section{References}

[1] N.U. Ahmed, X. Xiang, Nonlinear boundary control of semilinear parabolic systems, SIAM J. Control Optim. 34 (1996) 473-490.

[2] H. Amann, Parabolic evolution equations and nonlinear boundary conditions, J. Differential Equations 72 (1988) 201-269.

[3] A. Bensoussan, G.D. Prato, M.C. Delfour, S.K. Mitter, Representation and Control of Infinite Dimensional Systems, Vol. I, Birkhäuser, Boston, 1992.

[4] H. Bercovici, C. Foias, A. Tannenbaum, The structured singular value for linear input/output operators, SIAM J. Control Optim. 34 (1996) 1392-1404.

[5] J.B. Conway, A Course in Functional Analysis, Springer, New York, 1990.

[6] R.F. Curtain, H. Zwart, An Introduction to InfiniteDimensional Linear System Theory, Springer, New York, 1995.
[7] J.C. Doyle, Analysis of feedback systems with structured uncertainties, IEE Proc. Part D 192 (1982) 242-250.

[8] P. Gahinet, M. Sorine, A.J. Laub, G. Kenney, Stability margins and Lyapunov equations for linear operators in Hilbert space, Proceedings of the 29th IEEE Conference on Decision and Control, 1990, pp. 2638-2639.

[9] I. Gohberg, S. Goldberg, M.A. Kaashoek, Classes of Linear Operators, Vol. I, Birkhäuser, Boston, 1990.

[10] D. Hinrichsen, A.J. Pritchard, Real and complex stability radii: a survey, Proceedings of the Workshop Control of Uncertain Systems, Bermen, 1989, Progr. System Control Theory 6 (1990) 119-162.

[11] D. Hinrichsen, A.J. Pritchard, Robust stability of time-invariant infinite dimensional systems with respect to time-varying perturbations, Proceedings of the European Control Conference, 1993.

[12] D. Hinrichsen, A.J. Pritchard, Robust stability of linear evolution operators on Banach spaces, SIAM J. Control Optim. 32 (1994) 1503-1541.

[13] T. Kato, Perturbation Theory of Linear Operators, Springer, New York, 1966.

[14] J.L. Lion, E. Magenes, Non-Homogeneous Boundary Value Problems and Applications, Vol. I, Springer, New York, 1972.

[15] S.-H. Lu, I.-K. Fong, Stability radius of linear normal distributed parameter systems with multiple directional perturbations, Proceedings of the 37th IEEE Conference on Decision and Control, 1998, pp. 819-820.

[16] A. Pazy, On the applicability of Lyapunov's theorem in Hilbert space, SIAM J. Math. Anal. 3 (1972) 291-294.

[17] A. Pazy, Semigroups of Linear Operators and Applications to Partial Differential Equations, Springer, New York, 1983.

[18] A.J. Pritchard, S. Townley, Robustness of linear systems, J. Differential Equations 77 (1989) 254-286. 